\title{
Spatial interaction of methylene blue stained soil pores
}

\author{
A. Stein ${ }^{\mathrm{a}, *}$, M.N.M. Van Lieshout ${ }^{\mathrm{b}}$, H.W.G. Booltink ${ }^{\mathrm{c}}$ \\ a Department of Mathematics, Wageningen University, Dreijenlaan 4, \\ 6703 HA Wageningen, Netherlands \\ ${ }^{\mathrm{b}}$ CWI, P.O. Box 94079, 1090 GB Amsterdam, Netherlands \\ ${ }^{\mathrm{c}}$ Department of Environmental Sciences, Wageningen University, P.O. Box 37, \\ 6700 AA Wageningen, Netherlands
}

Received 3 April 2000; received in revised form 27 September 2000; accepted 30 October 2000

\begin{abstract}
In this paper, we compare different types of patterns that emerge when applying methylene blue dye as a tracer on soils to detect preferential flow paths as a result of large cracks. Patterns on channels, vughs and cracks are analyzed with the $J$-function and with indicator variograms. By means of a combined analysis, we obtained a better understanding of the patterns as well as some clear indications for precision agriculture management. The study is illustrated with methylene blue patterns obtained at two locations at four succeeding depths in the Netherlands. (c) 2001 Elsevier Science B.V. All rights reserved.
\end{abstract}

Keywords: Methylene blue; Stains; Spatial interaction

\section{Introduction}

The occurrence of large cracks and channels in soils, generally referred to as macropores, has a major impact on water and solute movement. A clear definition of a macropore is difficult to give. Beven and Germann (1982)

\footnotetext{
* Corresponding author.

E-mail addresses: alfred.stein@bodlan.beng.wau.nl, alfred.stein@wts.wk.wau.nl (A. Stein).
} 
summarized literature and concluded that macropore sizes varied in a wide range between 30 and $3000 \mu \mathrm{m}$. The effects of macropore systems on water and solute transport are highly variable and cannot be characterized by pore sizes only. A functional characterization is therefore required. For precision farming characterization of stains is of an increasing importance to better understand where water containing nitrate and pesticides is likely to reach the ground water and hence may cause risks for humans or the environment (Stein et al., 1997; Groffman, 1997). This contributes to detailing the concept of the sustainability concept in agriculture (Stein, 1994; Barnett et al., 1995; CIBA, 1997).

In soils, basically three different types of macropores occur: cracks, vughs and circular channels. For a better understanding of the three-dimensional structure of stains, a series of two-dimensional images taken at the same locations in space but at several succeeding depths can be useful. For those images, the classification is based upon the iso-perimetric ratio of $r=A / \rho^{2}$,were $A$ is the pore area and $\rho$ is the macropore perimeter. If $r<0.015$, the macropore is classified as a crack, if $0.015 \leq r<0.04$, then it is a vugh, and if $r \geq 0.04$, it is referred to as a circular channel (Bouma et al., 1977; RingroseVoase and Bullock, 1984). Circular channels, therefore, have a small perimeter as compared to their area and are closer to a circular shape, whereas cracks have a small area as compared to their perimeter and vughs are intermediate. Due to their shape and orientation cracks, vughs and channels have clearly different physical and chemical characteristics with respect to transport and absorption processes of soil moisture into soils. If a soil is dominated by large vertical continuous cracks, little lateral interaction exists between water in the cracks and the adjacent soil, with limited absorption as a result. Vughs or extremely large channels, on the other hand, are often horizontally oriented in which the lateral interaction is much larger. In these circumstances, the soil will absorb much more water and solutes.

In the past, fractal theory has been used successfully to quantitatively characterize macropore structure (Bartoli et al., 1991). Hatano and Booltink (1992) and Booltink et al., (1993) used fractal dimensions of methylene bluecolored macropores and related these to measured soil physical characteristics with the aim to predict macropore flow dynamically. Fractal theory, however, does not allow to characterize relations between different types of stains and other statistical procedures have to be applied instead.

Statistical studies of soil structure are rather limited so far. Bartoli et al. (1999) expressed pore size distributions as probability density functions with the aim to explain diffusion processes in the soil. This technique, however, is applicable only to some water and solute transport studies since only a small fraction of the total pore volume is actively conducting water. To overcome this, Stein et al. (1998) used the Kaplan-Meier estimate to quantify the structure of patterns of individual macropores of different types. In that study, they did not yet take into account the spatial interaction between different macropore types. 
On the other hand, point processes have been applied in the soils science literature, with a focus either on distribution of tree rooting spatial distributions (Pierret et al., 1999), on earthworm burrow systems (Chadoeuf et al., 1994; Daniel et al., 1997; Capowicz et al., 1998) or on pore distributions (Vogel and Kretzschmar, 1996) including applications to control strategy (Debras et al., 1998). However, only Chadoeuf et al. (1994) considered a quantitative analysis of spatial interactions seems.

Point pattern analysis (Van Lieshout and Baddely, 1997) can be useful to understand the interaction between cracks, vughs and circular channels. Interaction between patterns may elucidate origins of pattern. For example, one type of pattern (like channels) may occur predominantly in the neighborhood of other patterns (like that of cracks), whereas the inverse may not hold. In particular, we study the interaction between the vugh process and the other patterns to better explain their nature. Further, interactions may change with increasing depth, revealing the effects of processes that act from the top of the soil profile as compared to processes that are constant at various depths.

In a slightly dissimilar way, patterns of blue-colored stains on two-dimensional images can be used as a 0-1 variable in a bounded domain. Under the assumption of stationarity, which may hold at least locally, variograms can be constructed at each depth that an image was taken. Such a geostatistical analysis then allows to interpolate these variables towards depths where insufficient data were collected. As a result, maps can be obtained with probabilities where saturated moisture is most likely to occur and hence where a reduction in pesticide and nitrate application should be achieved. On the other hand, other parts may be of a limited vulnerability, as these can hardly be reached by any moisture spilled on the soil surface.

This study was conducted to demonstrate the use of different statistical pattern analysis techniques to model the spatial interaction between macropores that conduct methylene blue-colored water in a heavily textured structured clay soil. On one hand, we aim to model the variability, on the other hand, we highlight some support issues for management.

\section{The case study}

In a study carried out in 1991, methylene blue-colored water was spoiled over the soil surface at two sites of $0.2 \times 0.2 \mathrm{~m}^{2}$ taken from a single field (Hatano and Booltink 1992; Booltink et al., 1993). The sites were located in newly reclaimed land, which at that stage contained no earthworms. After 3 days, the soil was cut at horizontal slices at depths with increasing intervals of $0.02 \mathrm{~m}$. A window $W$ of size $0.2 \times 0.2 \mathrm{~m}$ was used with a pixel size of $250 \times 250 \mu \mathrm{m}$. 
Each pixel is either blue or white. The methylene blue patterns were photographed and later analyzed by an image analyzer (Nexus 6400). Bitmaps produced by the image analyzer were used for further analysis. Of each stain, the center of gravity as well as the classification into cracks, vughs and channels were determined using the SCILIMAGE (Ten Kate et al., 1990) program. The two sites are denoted by BR04 (Fig. 1) and BR06 (Fig. 2), respectively, and a roman numeral is added to indicate the depth in units of $2 \mathrm{~cm}$.

The soil was classified as a 'mixed mesic Hydric Fluvaquent' (Soil Survey Staff, 1992) with a dominating heavy clay texture. This soil type generally contains strongly weathered cracks and circular channels. Cracks are formed by structural shrinkage of the soil due to reclamation, or by soil tillage. Circular channels arise because of roots; little is known about the origin of vughs. Of interest in this study was quantification of the interaction between stains of different types, in order to gain a better understanding of the nature of the stains. In particular, whether vughs could be related to either cracks or channels on the basis of the observed spatial patterns. Since the stains almost disappear below 8 $\mathrm{cm}$ depth-where water and solutes are transported through a few large
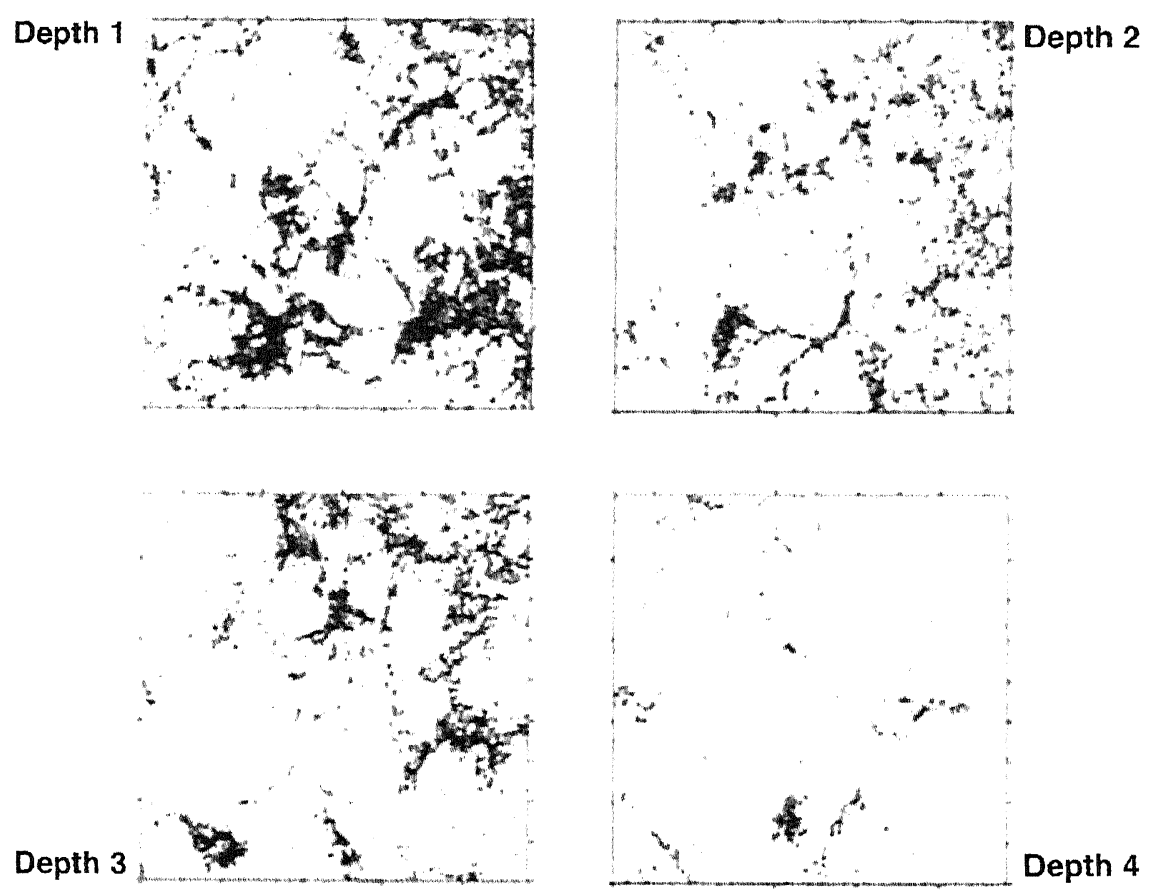

Fig. 1. First replicate, BR04, of methylene blue-colored stains at four depths separated by $2 \mathrm{~cm}$ below the soil surface. 

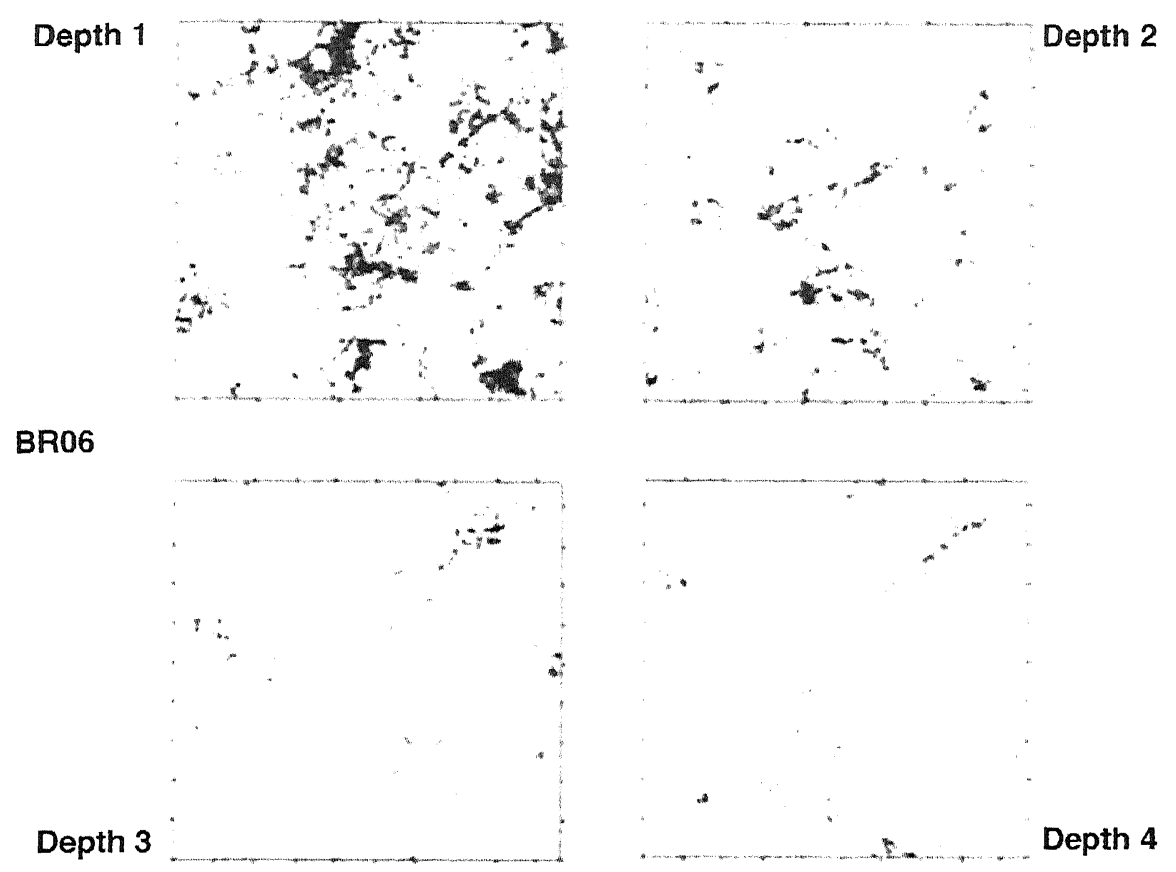

Fig. 2. Second replicate, BR06, of methylene blue-colored stains at four depths separated by $2 \mathrm{~cm}$ below the soil surface.

channels and interaction between soil and water is minimised-we restrict attention to the first four depths of the two replicates.

\section{Theory}

\subsection{Point patterns}

In the first part of the paper, the stains are represented by the coordinates of their center of gravity marked by type. As a result, each observed soil slice is conveniently summarised by three mapped point patterns (cf. Figs. 3 and 4) representing the cracks, vughs and channels. We shall assume that the sites and the slices are typical representatives of the soil surface from which they were taken as a whole. As each stain has a positive, non-null area, such representation of stains is an approximation only.

More formally, let $Y=\left(X_{1}, X_{2}, X_{3}\right)$ be a trivariate point process in $\mathbb{R}^{2}$ with jointly stationary components. The process consisting of all points regardless of type will be denoted by $X_{\bullet}=X_{1} \cup X_{2} \cup X_{3}$. In this paper, statistical inference for $Y$ will be based on distances, either those between a fixed reference point $y \in \mathbb{R}^{2}$ and the points of the process, or between the points of $X \bullet$ themselves. Alternatively, a second order approach could have been taken based on Ripley's 

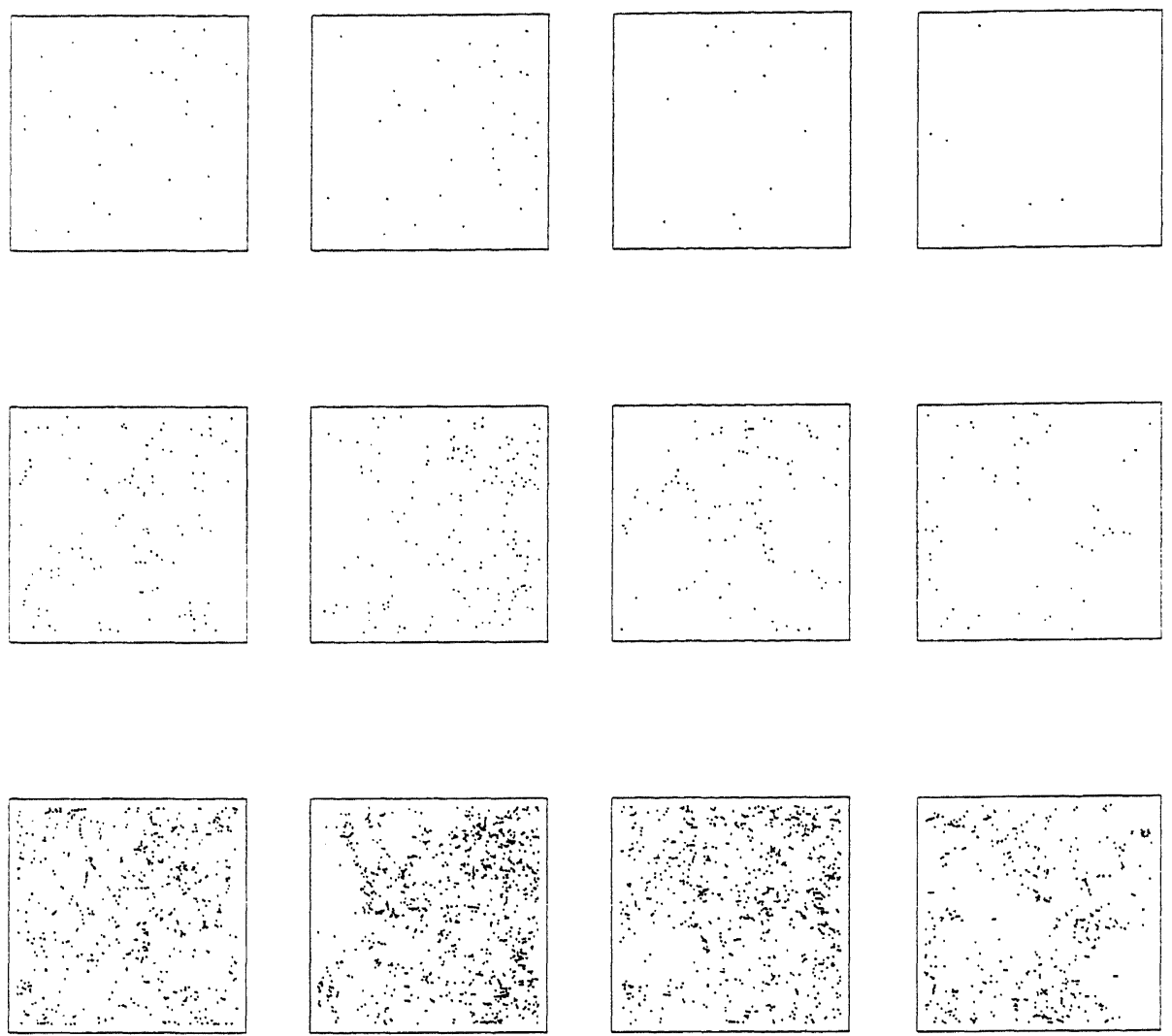

Fig. 3. Cracks (top row), vughs (middle row) and channels (bottom row) at four successive depths for the first replicate.

K-function (Ripley, 1977) or the pair correlation function (see, e.g. Stoyan et al., 1995).

Thus, for each $y \in \mathbb{R}^{2}$, let $\rho\left(y, X_{\bullet}\right)$ denote the distance from $y$ to the nearest stain. Then the empty space function of $X \bullet$ is defined for $r \geq 0$ as:

$$
F_{\bullet}(r)=\operatorname{Pr}\left\{\rho\left(y, X_{\bullet}\right) \leq r\right\},
$$

the probability of observing at least one stain closer than $r$ to $y$. Alternatively, the heuristic explanation of $1-F_{\bullet}(r)$ is the probability of the event that a randomly placed disk with radius $r$ does not contain a stain. The empty space function of $X_{i}, i \in\{1,2,3\}$, is denoted by $F_{i}$. By the stationarity assumption, $F_{\bullet}$, $F_{1}, F_{2}$ and $F_{3}$ do not depend on the choice of the reference point $y$.

Turning to inter-stain distances, the nearest neighbor distance function $G_{\bullet}$ is the distribution function of the distance from a typical stain to its nearest neighbor:

$$
G_{\bullet}(r)=P^{! y}\left\{\rho\left(y, X_{\bullet}\right) \leq r\right\}
$$



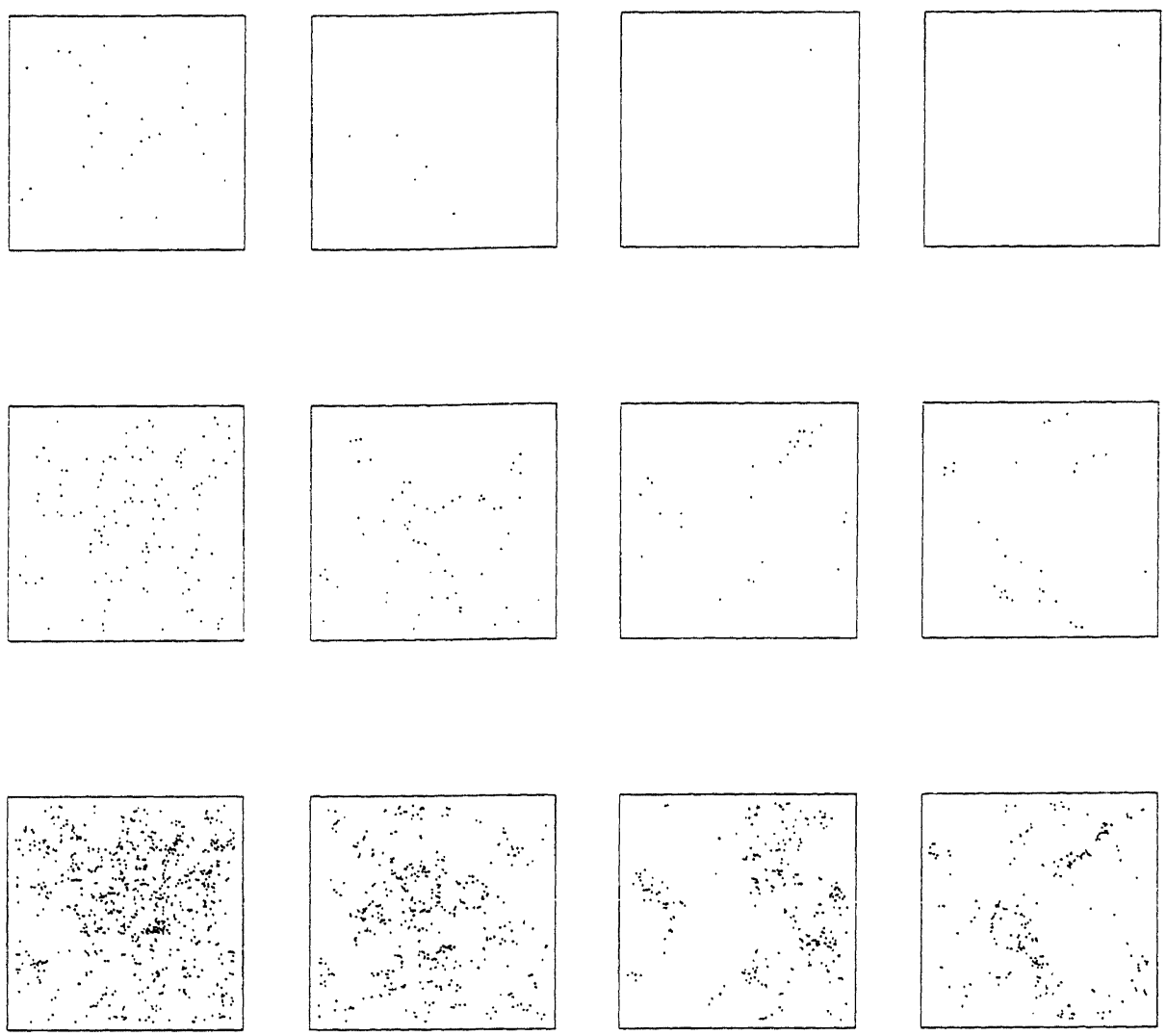

Fig. 4. Cracks (top row), vughs (middle row) and channels (bottom row) at four successive depths for the second replicate.

for $r \geq 0$. Here, $P^{! y}$ denotes the reduced Palm distribution of $X \bullet$ at $y \in \mathbb{R}^{2}$ (Cressie, 1991; Stoyan et al., 1995). The function $G \bullet(r)$ can be interpreted as the conditional distribution of the remainder of $X \bullet$ given a stain at $y$, i.e. as $\operatorname{Pr}\left\{\rho\left(y, X_{\bullet} \backslash\{y\}\right) \leq r \mid y \in X_{\bullet}\right\}$, where $X_{\bullet} \backslash\{y\}$ denotes the full process $X_{\bullet}$ from which the stain $\{y\}$ is excluded. A heuristic description of $1-G_{\bullet}(r)$ is the probability that a disk with radius $r$ centered on a randomly selected stain does not contain a further stain. Again, $G \bullet(r)$ does not depend on $y$ because of stationarity. The univariate nearest neighbor distance functions are denoted by $G_{i}(r), i \in\{1,2,3\}$.

Comparison of inter-stain distances to distances with respect to a reference point, say $X=0$, yields the $J$-function, defined by:

$$
J \bullet(r)=\frac{1-G \cdot(r)}{1-F_{\bullet}(r)}
$$

for all $r \geq 0$ satisfying $F_{\bullet}(r)<1$. The $J$-function is a useful index for the type and strength of spatial interaction. For Poisson processes (i.e. fully random 
processes without any clustering or regularity), the $J$-function is identically equal to 1 ; $J$-values larger than 1 are indicative of inhibition between the points, clustered patterns generally give rise to $J$-values smaller than 1 . Moreover, the $J$-function is constant beyond the effective range of interaction (Van Lieshout and Baddeley, 1999).

In this study, we are primarily interested in the interactions between the components. Thus, the cross versions of the above functions are needed. Write $P^{!\{y, i\}}$ for the reduced Palm distribution of $Y$ conditional on a stain of type $i$ at a point $y$. Then the $i$-to- $j$ nearest neighbor distance function $G_{i j}(r)$ is defined as:

$$
G_{i j}(r)=P^{\{\{y, i\}}\left\{\rho\left(y, X_{j}\right) \leq r\right\} .
$$

Again, $G_{i j}(r)$ does not depend on the choice of $y$. Thus, $G_{i j}(r)$ is the distribution function of the distance from a typical stain of type $i$ to the nearest stain of type $j$.

Finally, for $i \neq j$, set:

$$
J_{i j}(r)=\frac{1-G_{i j}(r)}{1-F_{j}(r)}
$$

for all $r \geq 0$ for which $F_{j}(r)<1$. Thus, the $i$-to- $j J$-function compares the distribution of distances to the nearest stain of type $j$ from respectively a typical type $i$ stain and the origin. As such, it can be seen as a measure of correlation between $X_{i}$ and $X_{j}$. If $X_{i}$ and $X_{j}$ are (marginally) independent, $J_{i j}(r) \equiv 1$. Values $J_{i j}(r)>1$ can be interpreted as indicating inhibition of type $j$ stains by type $i$ stains; indeed $J_{i j}(r)>1$ is equivalent to $G_{i j}(r)<F_{j}(r)$, that is, the presence of a type $i$ stain decreases the probability of finding a type $j$ stain close by. Similarly, values less than 1 suggest positive association, i.e. attraction of stains of type $j$ by a stain of type $i$. For more details, see Van Lieshout and Baddeley (1997, 1999).

\subsection{Geostatistical interpolation}

A single image shows horizontal variability, whereas a sequence of images at different depths shows variability in depth as well. Characterization of two-dimensional spatial variability can be useful for modeling interactions and understanding the processes in soils, whereas analysis of three-dimensional variability may help to predict patterns emerging at unobserved depths and as such may indicate occurrence of connecting stains. Variability in depth differs from horizontal, planar variability. In particular, roots grow from the soil surface towards the ground water, causing a clear non-stationarity of stains, both in number, in size and in shape with depth. In this section, we decided to model variability in depth separately from planar variability. An interesting example on modelling three-dimensional dependence by means of anisotropy is given in Dagan (1989). We will now turn towards a geostatistical description of three-di- 
mensional variability, and we will consider images at various depth successively.

Consider a variable $\mathbf{y}(\mathbf{s}, d) \in \mathbb{R}$ with $\mathbf{s} \in \mathscr{D} \subset \mathbb{R}^{2}$ and $d \in \mathbb{R}$. In principle, any regionalized variable can be considered, but we will focus on a variable that takes the values 1 at blue-colored locations and the value 0 at uncolored locations. Observations are made at multiple locations in several depths. Let the depths be denoted by $d_{a}, a=1, \ldots, k$. At depth $d_{u}$, observations $\mathbf{y}\left(\mathbf{s}_{i a}, d_{a}\right)$ are made at $N_{a l}$ locations $\mathbf{s}_{i a}, i=1, \ldots, N_{a}$ at the image, where $\mathbf{s}_{i a}=\left(s_{i a x}, s_{i a y}\right)$ with $s_{i a x}$ and $s_{i a y}$ denoting the coordinates along two axes. This section discusses how the concept of variogram can be modified to accommodate three-dimensional data. The space-depth covariogram and the space-depth generalized covariance function can be treated in a similar way (Serra, 1982; Matheron, 1973).

Let $d_{a}$ be any depth and let two points $P_{1}$ and $P_{2}$, with coordinates $\left(\mathbf{s}, d_{a}\right) \in \mathscr{D} \times \mathbb{R}$ and $\left(\mathbf{s}+\mathbf{h}_{2}, d_{a}\right) \in \mathscr{D} \times \mathbb{R}$ be separated from each other by a distance $\mathbf{h}$ in space. For $\mathbf{y}\left(\mathbf{s}, d_{a}\right)$ we assume the intrinsic hypothesis to apply in the plane, i.e. $E\left(\mathbf{y}\left(\mathbf{s}+\mathbf{h}, d_{a}\right)-\mathbf{y}\left(\mathbf{s}, d_{a}\right)\right)=0$ for all $\mathbf{h}$. The 2-D variogram at depth $d_{a}$ is defined as:

$$
\gamma_{d_{a}}(r)=\frac{1}{2} E\left(\mathbf{y}\left(\mathbf{s}+\mathbf{h}, d_{a}\right)-\mathbf{y}\left(\mathbf{s}, d_{a}\right)\right)^{2}
$$

where $r=|\mathbf{h}|=\sqrt{|\mathbf{h}|^{2}}$. The 2-D variogram describes the horizontal variation as a function of the horizontal distance at depth $d_{a}$. A variogram estimator for this model is obtained by replacing the expectation by the average value of the squares of the squares of the differences of the observations:

$$
\hat{\gamma}_{d_{l a}}(\mathbf{h})=\frac{1}{2 N(\mathbf{h})} \sum_{i=1}^{N(h)}\left(\mathbf{y}\left(\mathbf{s}_{i a}+\mathbf{h}, d_{a}\right)-\mathbf{y}\left(\mathbf{s}_{i a}, d_{a l}\right)\right)^{2} .
$$

Although this may appear to be restrictive at first sight, it allows to treat variograms which change over depth.

A common activity in geostatistics is spatial interpolation by means of kriging. In what we call three-dimensional depth-slice kriging we aim to predict the value of $\mathbf{y}\left(\mathbf{s}_{0}, d_{0}\right)$, in particular if $\left(\mathbf{s}_{0}, d_{0}\right)$ does not coincide with an observation point. For this procedure, multiple observations are required at a few depths. Kriging is carried out with a two-step procedure. In the first step, for any depth $d_{c}$, the value in the point with coordinate vector $\mathbf{s}_{0}$ is predicted, being the points with the same spatial coordinates as those of the prediction location but at each of the $k$ depths. To make these predictions, estimated 2-D variograms and observations at depth $d_{a}$ are used. This yields predictors $\hat{\mathbf{y}}\left(\mathbf{s}_{0}, d_{a}\right)=$ $\sum \lambda_{i a} \mathbf{y}\left(\mathbf{s}_{i a}, d_{a}\right)$ for $a=1, \ldots, k$ with kriging weights $\lambda_{i a}$ and kriging variance $\operatorname{Var}\left(\hat{\mathbf{y}}\left(\mathbf{s}_{0}, t_{a}\right)-\mathbf{y}\left(\mathbf{s}_{0}, t_{a}\right)\right)$. Also, the variance of the predictor $\hat{\mathbf{y}}\left(\mathbf{s}_{0}, d_{a}\right)$ is obtained, which equals $\sigma_{a}^{2}=\operatorname{Var}\left(\sum_{i=1}^{N_{a}} \lambda_{i a} \mathbf{y}\left(\mathbf{s}_{i a}, d_{a}\right)\right)=\lambda^{(a) \prime} \mathbf{C} \lambda^{(a)}$ where $\boldsymbol{\lambda}^{(a)}$ is the vector 
of kriging weights at $d_{a}$. The variance of the predictor differs from the more commonly applied kriging variance, which is the variance of the prediction error.

At the second step, the value of $\mathbf{y}\left(\mathbf{s}_{0}, d_{0}\right)$ at the prediction point $\left(\mathbf{s}_{0}, d_{0}\right)$ is predicted, using the $k$ predictions obtained in the first step. The predictor $u$, linear in the predictors $\hat{\mathbf{y}}\left(\mathbf{s}_{0}, d_{a}\right)$, equals:

$$
u=\sum_{a=1}^{k} \boldsymbol{\vartheta}_{\alpha} \hat{\mathbf{y}}\left(\mathbf{s}_{0}, d_{a}\right)=\sum_{a=1}^{k} \sum_{i=1}^{N_{a}} \boldsymbol{\vartheta}_{a} \lambda_{i a} \mathbf{y}\left(\mathbf{s}_{i a}, d_{a}\right)
$$

where $\boldsymbol{\vartheta}$ is a vector of kriging weights with a similar interpretation as the kriging weights $\lambda_{i a}$ obtained before, but now obtained for the one-dimensional depth direction. If the observations can be considered to be independent in depth, the kriging variance is given by:

$$
\operatorname{Var}\left(u-\mathbf{y}\left(\mathbf{s}_{0}, d_{0}\right)\right)=\sigma_{0}^{2}+\boldsymbol{\vartheta}_{1}^{2} \sigma_{1}^{2}+\ldots+\boldsymbol{\vartheta}_{k}^{2} \sigma_{k}^{2}
$$

where $\sigma_{0}^{2}$ is the variance at $d_{0}$. Therefore, the variances of the predictors contribute with squared kriging weights, and the variance $\sigma_{0}^{2}$ is added. An estimate for $\sigma_{0}^{2}$ can be obtained by summing the values of nugget variances and range calculated using the parameter estimates for $d_{0}$, for example by means of a (non-)linear regression. In depth, non-stationarity may be present. This can be taken into account by specifying in depth a polynomial trend.

The predictor thus obtained has some attractive properties. It is exact: values observed in observation locations are predicted exact and the kriging variance is equal to 0 . It honors changing spatial variability in depth. Data from several depths may be used, hence increasing the amount of information.

\section{Results}

\subsection{Point patterns}

Throughout this section we will refer to the cracks as pattern $X_{1}$, vughs as $X_{2}$ and channels as $X_{3}$. Descriptive statistics are given in Table 1 . At all depths channels are the most abundant stains in the two replicates, followed by vughs and cracks. Replicate BR04 contains more stains than replicate BR06, for which the number of cracks reduces to 1 at larger depths, i.e. for BR063 and BR064. Notice though that this single crack occurs at a place where it was not observed at the two shallowest depths, labeled here BR061 and BR062. Also the mean infiltrating area and the mean perimeter is much larger for BR04 than for BR06. The total area of cracks is much larger than that for vughs and channels, indicating that these form the predominant channels for leaching of contaminated moisture towards the groundwater. 
Table 1

Descriptive statistics of the cracks $\left(X_{1}\right)$, vughs $\left(X_{2}\right)$ and channels $\left(X_{3}\right)$ at four depths of the replicates

\begin{tabular}{|c|c|c|c|c|c|c|c|c|c|c|}
\hline & \multirow[t]{2}{*}{ Stain* } & \multicolumn{4}{|l|}{ Area } & \multicolumn{4}{|c|}{ Perimeter } & \multirow[t]{2}{*}{ No. } \\
\hline & & Mean & Std & Min & $\operatorname{Max}$ & Mean & Std & Min & $\operatorname{Max}$ & \\
\hline \multirow[t]{3}{*}{ BR041 } & $X_{1}$ & 1327 & 3056 & 25 & 15,212 & 382 & 570 & 42.3 & 2744 & 30 \\
\hline & $X_{2}$ & 43.6 & 45.0 & 5 & 303 & 37.2 & 21.8 & 11.8 & 132 & 119 \\
\hline & $x_{3}^{-}$ & 5.5 & 11.5 & 1 & 226 & 7.2 & 6.3 & 2.0 & 64.9 & 577 \\
\hline \multirow[t]{3}{*}{ BR042 } & $X_{1}$ & 379 & 403 & 46 & 2117 & 20.5 & 138.3 & 58.0 & 689.6 & 35 \\
\hline & $X_{2}$ & 40.3 & 38.7 & 4 & 295 & 36.0 & 19.7 & 10.8 & 137.8 & 158 \\
\hline & $x_{3}^{-}$ & 6.0 & 9.7 & 1 & 125 & 7.8 & 6.2 & 2.0 & 44.5 & 920 \\
\hline \multirow[t]{3}{*}{ BR043 } & $X_{1}$ & 1480 & 1915 & 75 & 7194 & 587.1 & 775.5 & 72.4 & 2973 & 14 \\
\hline & $X_{2}$ & 42.1 & 46.5 & 6 & 274 & 36.7 & 22.4 & 12.3 & 124.9 & 97 \\
\hline & $X_{3}$ & 5.3 & 7.3 & 1 & 72 & 7.3 & 5.7 & 2.0 & 41.2 & 760 \\
\hline \multirow[t]{3}{*}{ BR044 } & $X_{1}$ & 327.2 & 375.5 & 89 & 1062 & 151.8 & 76.3 & 87.7 & 280 & 6 \\
\hline & $X_{2}$ & 34.0 & 39.5 & 4 & 199 & 32.0 & 19.8 & 11.0 & 96.6 & 56 \\
\hline & $X_{3}$ & 4.50 & 5.5 & 1 & 54 & 6.8 & 5.0 & 2.0 & 36.1 & 458 \\
\hline \multirow[t]{3}{*}{ BR061 } & $X_{1}$ & 582.9 & 825.0 & 34 & 3490 & 238 & 205.3 & 48.3 & 938 & 29 \\
\hline & $X_{2}$ & 61.0 & 156.0 & 6 & 1634 & 39.6 & 32.9 & 12.3 & 285.8 & 123 \\
\hline & $X_{3}$ & 5.4 & 8.8 & 1 & 137 & 7.2 & 6.1 & 2.0 & 57.3 & 755 \\
\hline \multirow[t]{3}{*}{ BR062 } & $X_{1}$ & 363.4 & 272 & 86 & 675 & 185.3 & 106.1 & 85.2 & 358 & 5 \\
\hline & $X_{2}$ & 61.1 & 57.0 & 6 & 296 & 44.7 & 24.1 & 13.5 & 120.3 & 60 \\
\hline & $X_{3}^{-}$ & 5.3 & 9.1 & 1 & 130 & 7.1 & 6.1 & 2.0 & 56.5 & 412 \\
\hline \multirow[t]{3}{*}{ BR063 } & $x_{1}$ & 264 & & 264 & 264 & 134.0 & & 134 & 134 & 1 \\
\hline & $X_{2}$ & 47.2 & 34.4 & 9 & 154 & 39.3 & 16.2 & 15.6 & 73.8 & 26 \\
\hline & $\overline{X_{3}}$ & 4.3 & 6.1 & 1 & 55 & 6.1 & 5.5 & 2.0 & 36.7 & 278 \\
\hline \multirow[t]{2}{*}{ BR064 } & $X_{1}$ & 187 & & 187 & 187 & 144.4 & & 144.4 & 144 & 1 \\
\hline & $X_{2}$ & 27.9 & 31.6 & 8 & 154 & 29.1 & 18.3 & 14.4 & 93.8 & 31 \\
\hline
\end{tabular}

Our spatial analysis begins with examining each stain pattern separately. As the empty space and nearest neighbor distance functions depend on stain intensity, it is not easy to compare the replicates at various locations and depths. For the $J$-function, on the other hand, qualitative comparisons are quite straightforward, since for all Poisson processes regardless of the intensity $J(r) \equiv 1$.

In Fig. 5, the estimated univariate $J$-functions are plotted for the first replicate BR04 (dashed lines). To do so, we computed estimates $\hat{F}_{i}$ and $\hat{G}_{i}$ for the empty space and nearest neighbor distance functions and set $\hat{J}_{i}=(1-$ $\left.\hat{G}_{i}\right) /\left(1-\hat{F}_{i}\right)$. Since the data are collected over a bounded window only, edge effects have to be taken into account. Here we took the Kaplan-Meier (Kaplan and Meier, 1958) approach of Baddeley and Gill (1998) as advocated in Baddeley (1999). To explain the idea, consider the nearest neighbor distance function. For each point $x_{j} \in X_{1}$, say, its distance to the nearest other point of $X_{1}$ is censored by the boundary $\partial W$ of the observation window. Write $s_{j}=$ $\rho\left(x_{j}, W \cap X_{1} \backslash x_{j}\right)$ for the observed nearest neighbor distance and $b_{j}=\rho\left(x_{j}, \partial W\right)$ 

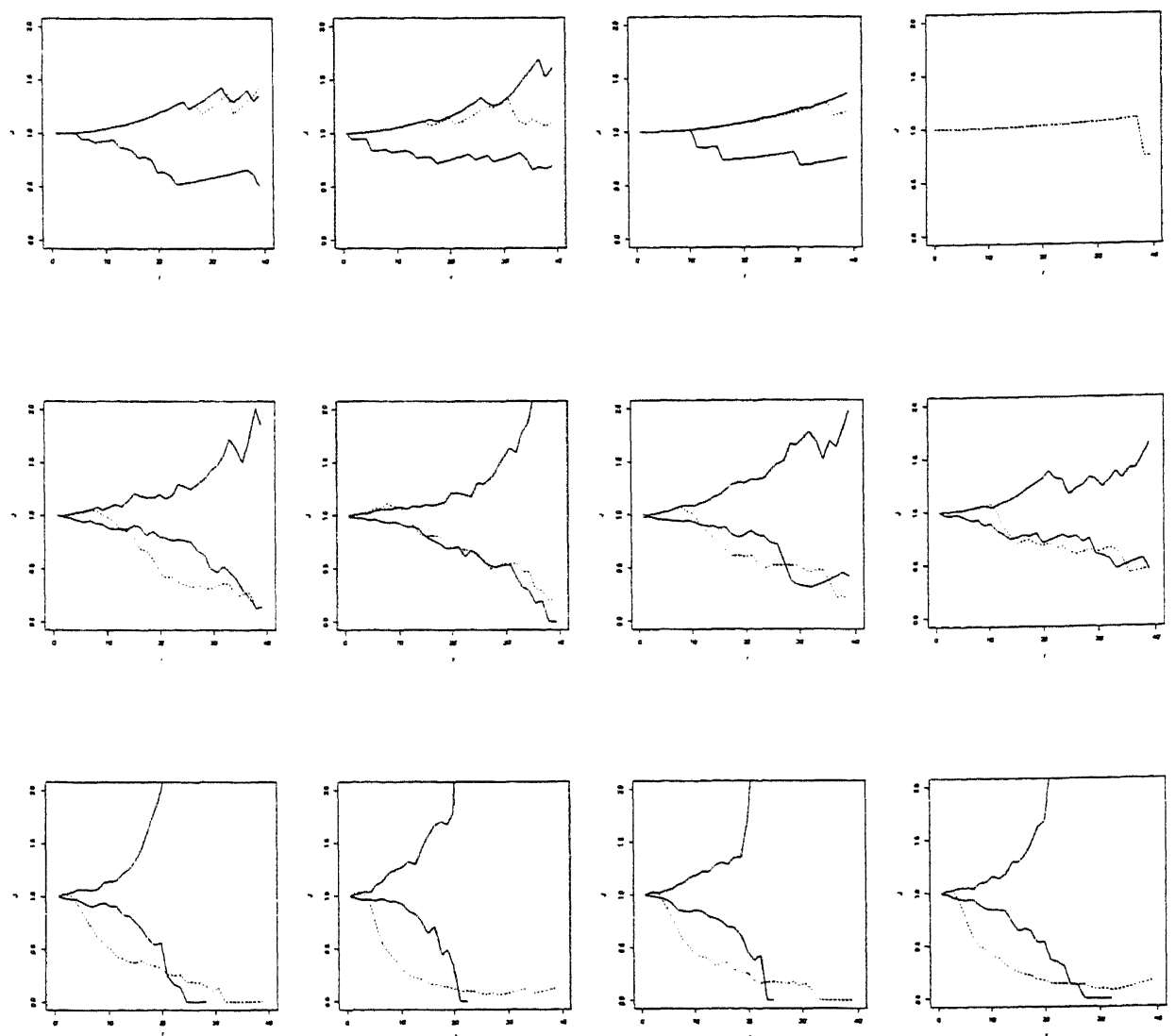

Fig. 5. Univariate $J$-functions of the patterns of cracks (top row), vughs (middle row) and channels (bottom row) at four successive depths for the first replicate.

for the distance to the boundary of $W$. Clearly $s_{j}$ is the true nearest neighbor distance for $x_{j}$ if $s_{j} \leq b_{j}$, and the Kaplan-Meier estimator is defined as:

$$
1-\hat{G}_{1}(r)=\prod_{s \leq r}\left(1-\frac{\#\left\{j: s_{j}=s ; s_{j} \leq b_{j}\right\}}{\#\left\{j: s_{j} \geq s ; s \leq b_{j}\right\}}\right) .
$$

Replacing the $s_{j}$ by between-type distances, similar formulae hold for the cross nearest neighbor distance functions. For the empty space function, additionally, a continuity correction is necessary, see Baddeley and Gill (1998) for further details. It can be seen that $J_{1}$ takes values somewhat above 1, suggesting mild inhibition. In contrast, $J_{2}$ and $J_{3}$ first increase, then decrease below 1 , indicating overall aggregation combined with inhibition at small range (most likely due to the physical size of the stains).

To see whether the deviations from spatial randomness are statistically significant at the 5\% level, 19 independent samples from Poisson processes with 
the same number of points as the data components were generated, and their $J$-functions estimated. The maximal and minimal values in the sample are recorded (Fig. 5, solid lines) and compared to the data estimates. Note that for the cracks - with the exception of BR044, which does not contain enough points to allow for meaningful results-the observed $J_{1}$ graph is mostly contained within the upper and lower envelopes, hence the inhibition is not significant. For the vughs on the other hand, the observed values lie mostly outside of the simulation envelopes, the deviation from randomness being even more significant for the channels.

The second replicate BR06 (Fig. 6) shows a similar pattern. Only the first depth BR061 contains a sizeable number of cracks; any suggestion of inhibition is not significant at the $5 \%$ level. The vugh and channel patterns display significant clustering, with inhibition at small range.
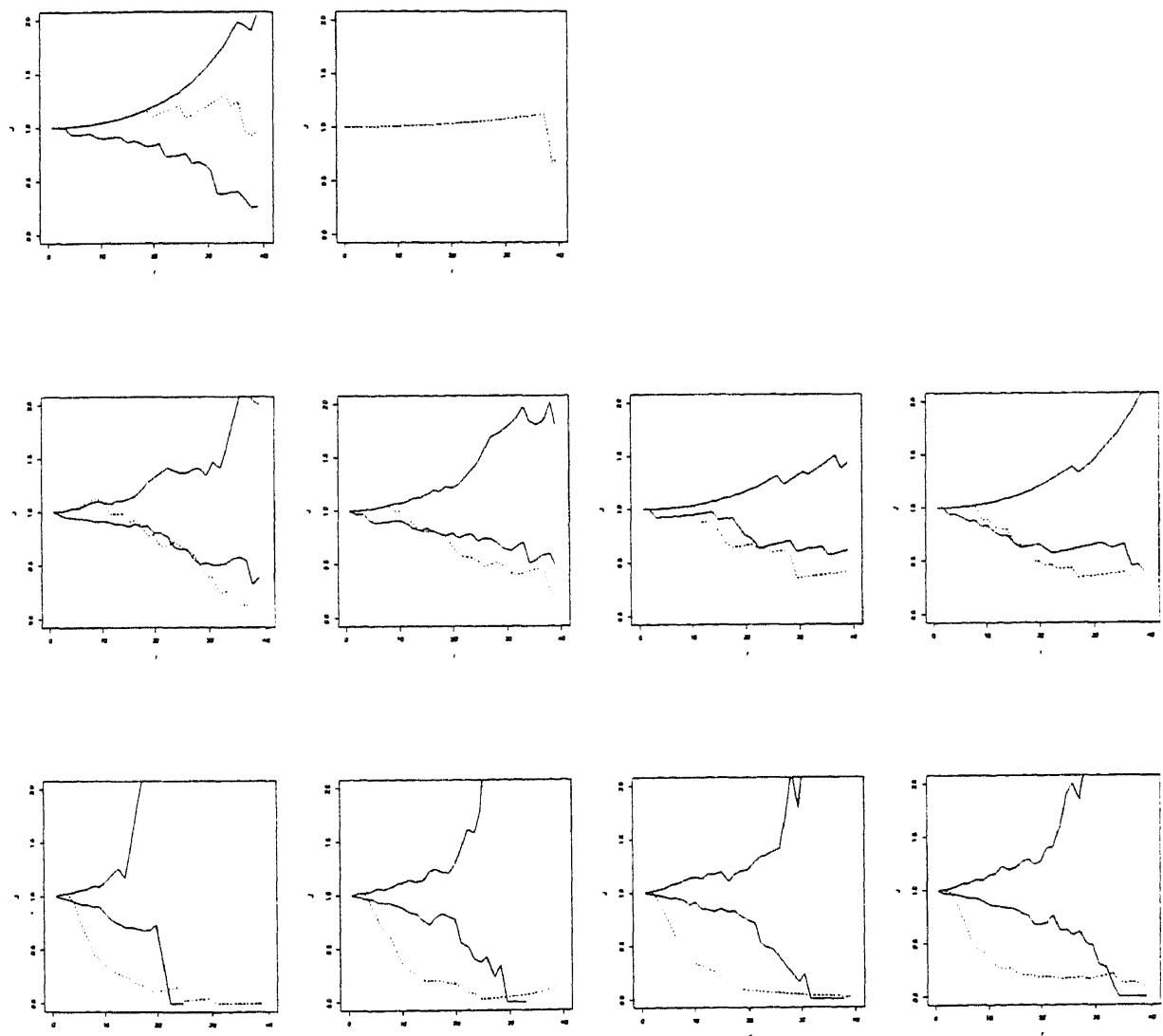

Fig. 6. Univariate $J$-functions of the patterns of cracks (top row), vughs (middle row) and channels (bottom row) at four successive depths for the second replicate. 
Next, we study whether the component patterns affect each other. First consider the vugh and channel components of the first replicate BR04. From the estimated cross $J$-functions (Fig. 7), we notice positive association between the vugh and channel patterns, with negative correlation at small range. To test whether the results are compatible with the hypothesis that $X_{2}$ and $X_{3}$ are independent, following Lotwick and Silverman (1982), opposite sides of the observation window are identified to obtain a torus, and one component pattern is translated randomly over this torus. For 19 independent torus translations, the relevant cross $J$-function is estimated, yielding upper and lower envelopes as before. As the cross $J$-functions estimated from the data fall outside of the simulation envelopes, the independence hypothesis is rejected at the $5 \%$ level. For the second replicate, analogous results are obtained (Fig. 8).

Turning to the interaction of the cracks with the other components, Fig. 9 plots estimates of $J_{1 i}$ (top row) and $J_{i 1}$ (bottom row) for BR041 and $i \in\{2,3\}$. We notice inhibition of vughs by cracks, as the estimated $J_{12}$-function is larger than 1 . The dependence of channels on cracks is more ambiguous. The presence of either vughs or channels does not seem to influence the crack pattern much, since the estimates of $J_{21}$ and $J_{31}$ are rather flat. The assumption that $X_{1}$ and $X_{2}$ are independent is rejected at the 5\% level in favor of inhibition for small ranges; the hypothesis that $X_{1}$ and $X_{3}$ are independent cannot be rejected. Regarding BR061, again vughs and channels do not seem to have a strong effect on the crack locations, while the plots of the estimated $J_{12^{-}}$and $J_{13}$-functions suggest mild inhibition of vughs and channels by cracks (see Fig. 10). Especially for the second replicate, the number of cracks at larger depths is rather small, so we prefer to consider only vughs and channels at these depths.
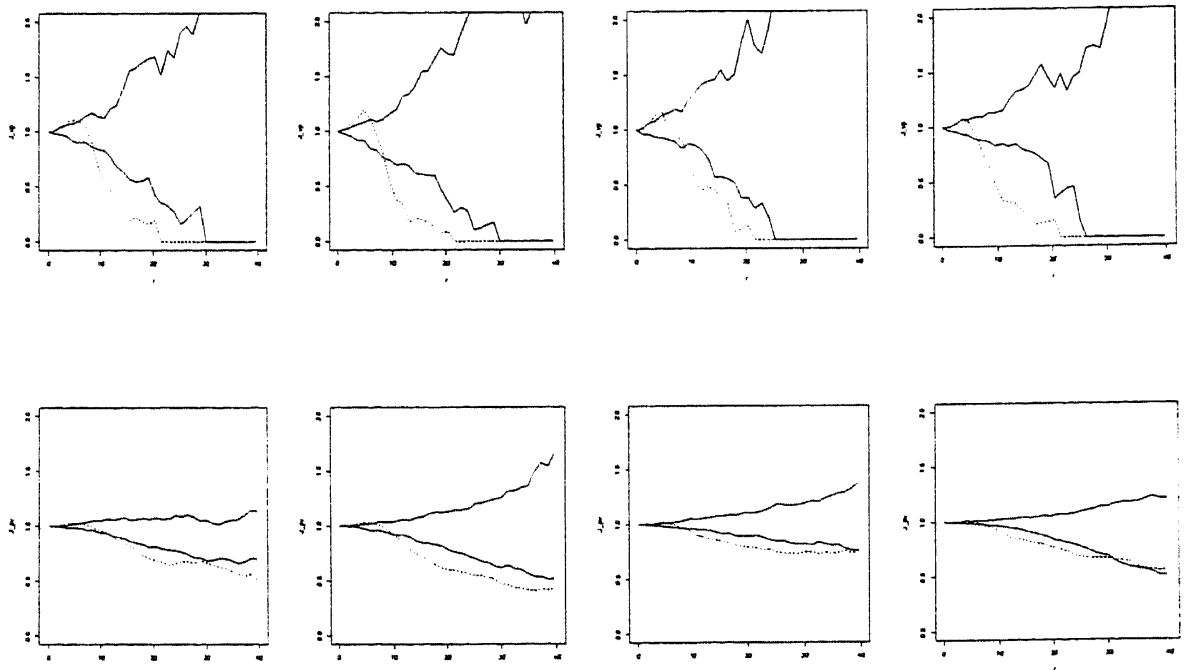

Fig. 7. Cross $J$-functions of channels with respect to vughs (top row) and vughs with respect to channels (bottom row) at four successive depths for the first replicate. 

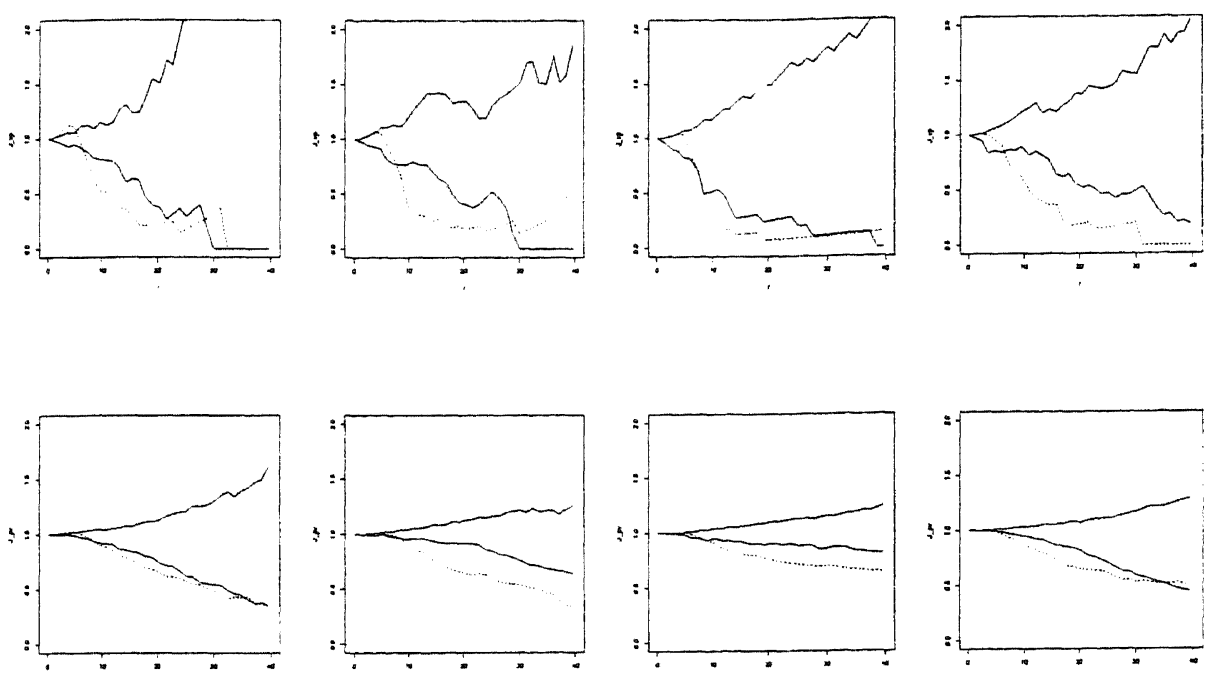

Fig. 8. Cross J-functions of channels with respect to vughs (top row) and vughs with respect to channels (bottom row) at four successive depths for the second replicate.

Note that above results are qualitatively similar for various depths and sites, supporting our assumption of stationarity.
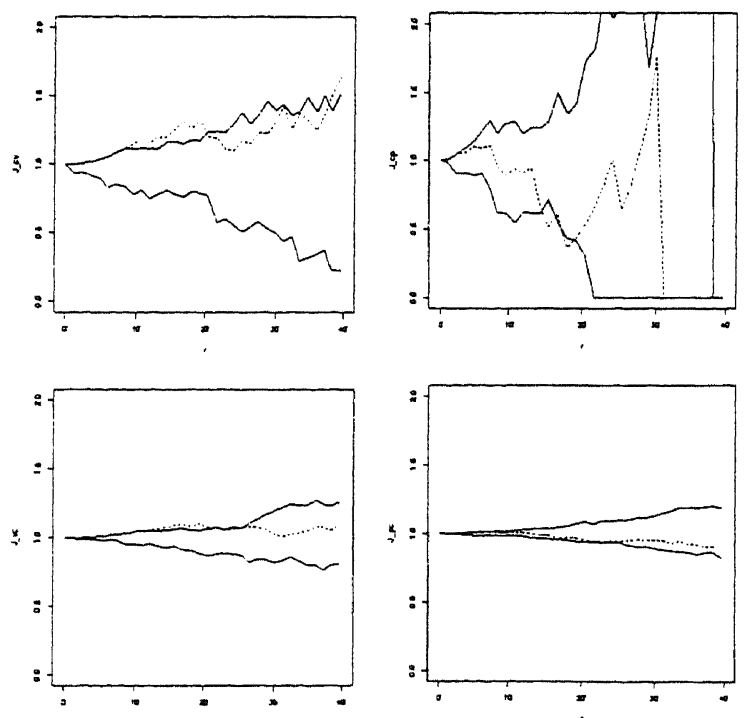

Fig. 9. Cross $J$-functions of vughs with respect to cracks (top left), channels with respect to cracks (top right), cracks with respect to vughs (bottom left) and cracks with respect to channels (bottom right) for the first depth of the first replicate. 

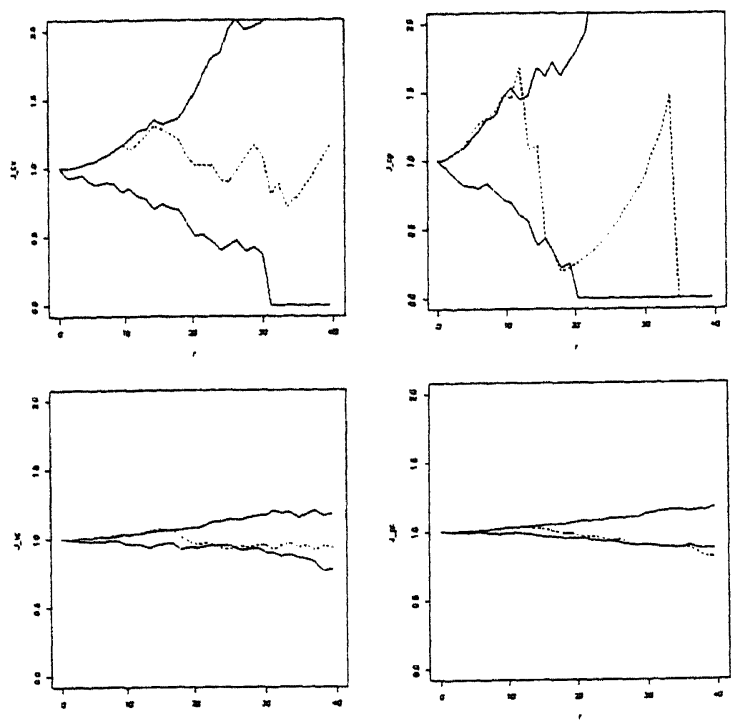

Fig. 10. Cross $J$-functions of vughs with respect to cracks (top left), channels with respect to cracks (top right), cracks with respect to vughs (bottom left) and cracks with respect to channels (bottom right) for the first depth of the second replicate.

\subsection{Geostatistical interpolation}

For the geostatistical interpolation, i.e. to create an image at those depths where no pictures were taken, we took a random selection of 1000 pixels locations from each of the three images. We estimated variograms in space (Table 2). An exponential model fitted well at the BR04 site, and also at the two upper depths of the BR06 site, whereas a pure nugget effect emerged at the third and fourth depth of BR06. As the range parameter is low (1 pixel and less), the patterns also at the BR04 site are strongly similar to a pure nugget effect.

We constructed interpolated images for both plots at depths equal to 0.03 , 0.05 and $0.07 \mathrm{~m}$ below the soil surface (Figs. 11 and 12). We notice that, in

Table 2

Estimated parameters of an exponential variogram model $\gamma(h)=C_{0}+C(1-\exp (-h / a))$

\begin{tabular}{llllc}
\hline & Depth & $C_{0}$ & $C$ & $a$ \\
\hline BR04 & 1 & 0.0982 & 0.063 & 54.1 \\
& 2 & 0.075 & 0.023 & 1.05 \\
& 3 & 0.029 & 0.096 & 0.48 \\
BR06 & 4 & 0.075 & 0.115 & 0.84 \\
& 1 & 0.059 & 0.046 & 28.5 \\
& 2 & 0.016 & 0.017 & 37.6 \\
& 3 & 0.011 & &
\end{tabular}




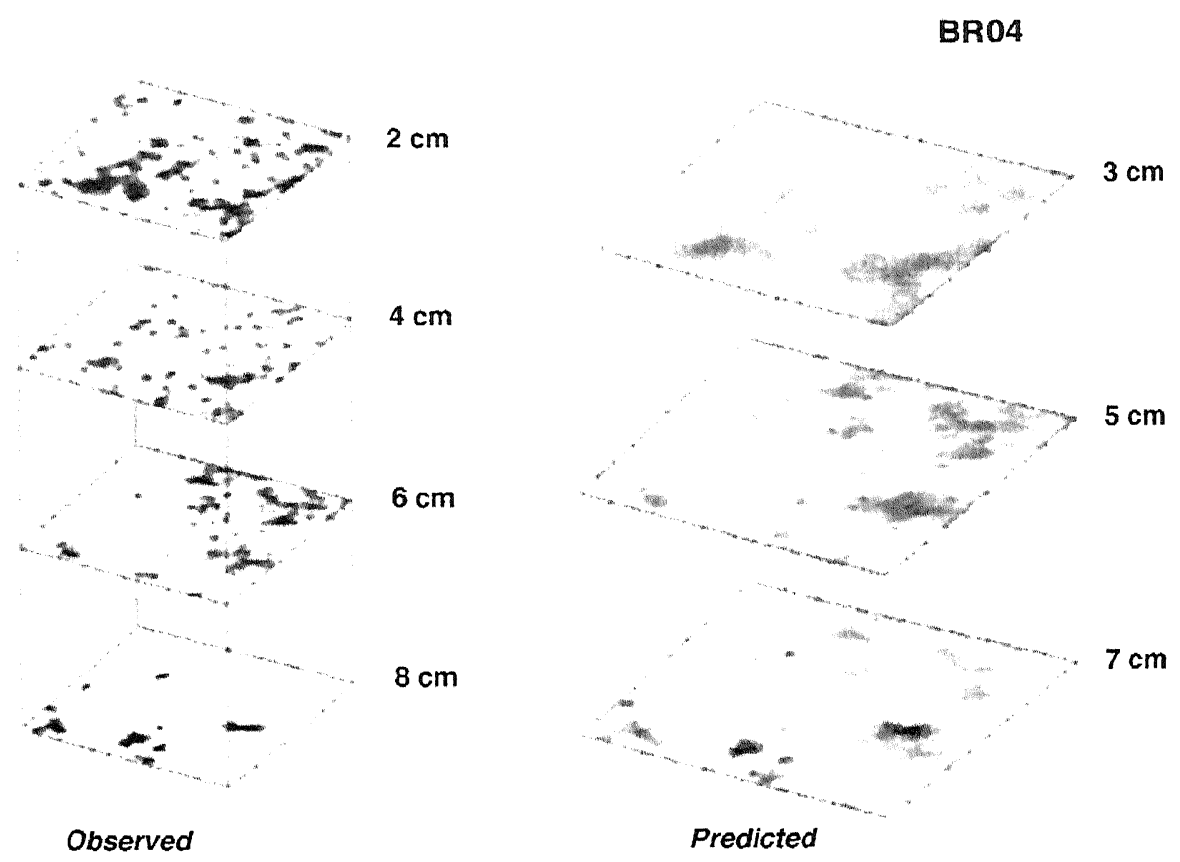

Fig. 11. Interpolated images at intermediate depths of $0.03,0.05$ and 0.07 below the soil surface for the first replicate.

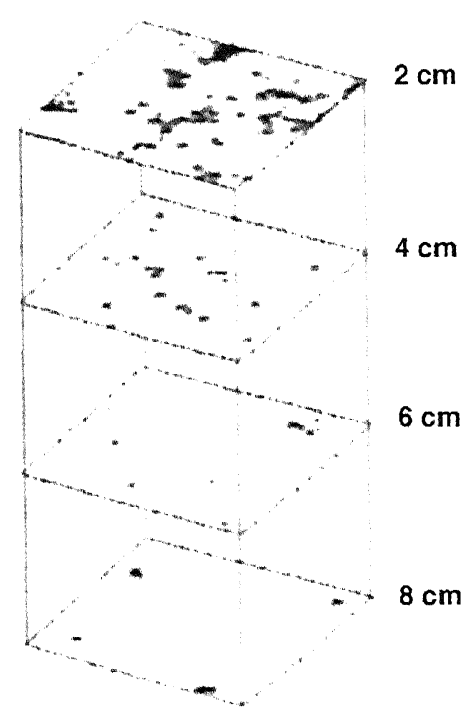

Observed

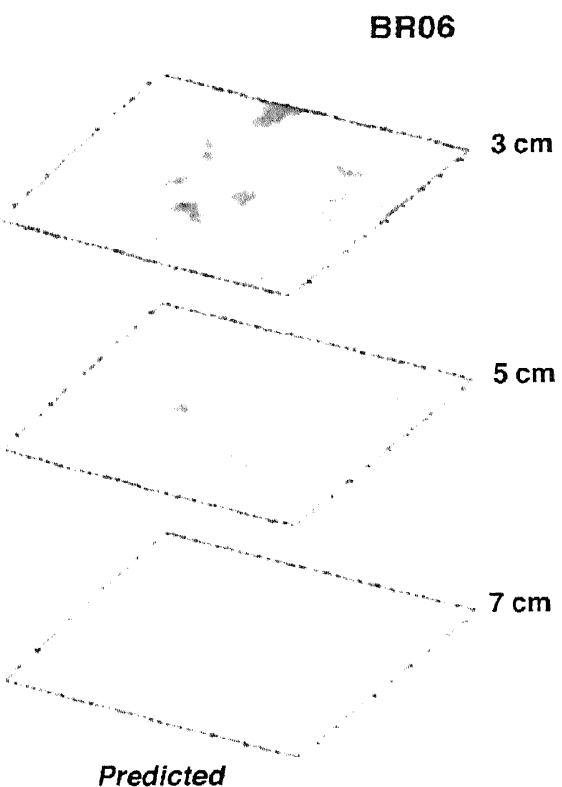

Fig. 12. Interpolated images at intermediate depths of $0.03,0.05$ and 0.07 below the soil surface for the second replicate. 
general, the images well correspond to the images at depths just above and just below the prediction depths, but that the pattern appears to be somewhat smoother than the original images. This is probably due to uncertainty caused by interpolation, where not only values equal to 0 and 1 are predicted, but also intermediate values may occur. But the sharp blue values in the lower left corners of BR04, depth 6 and $8 \mathrm{~cm}$ are well reproduced at the predicted depth 7 $\mathrm{cm}$ image. Also, the presence of strains at $6 \mathrm{~cm}$ and their absence at $8 \mathrm{~cm}$ depth is reflected in a bluey haze at depth 7 , indicating that the probability of the occurrence of stains is now equal to approximately 0.5 . Similar remarks apply to BR06.

\section{Discussion}

Identification of the different stains was done by means of point pattern analysis. Obviously, therefore, the stains were reduced to objects of dimension zero. As in practice, all stains have a positive area, and no two stains occur within each other, i.e. no channel can be identified within a crack, which is also physically impossible, a small inhibition for small distances inevitably occurs. This analysis also will not reveal the full connected pore space, and as such, preclude consideration of spatially random processes such as diffusion or dispersion, which are central for studies of aeration or reactive solute transport. Probably, stereological analysis could be useful here. It will also not provide information on moisture distribution. It does, however, provide ways of analyzing images of methylene blue-colored soil stains, to investigate relations between patterns and provide some ways of analyzing what occurred in between succeeding images.

A spatial point patterns analysis differs from a geostatistical analysis in several respects. First, a point pattern analysis refers to various patterns that can be related to each other. Interaction between various types of stains can be identified, quantified and tested for significance. A geostatistical analysis focuses entirely on an occurring pattern as such, and did not consider different forms of stains. It allows, though, to interpolate occurrence of blue-colored spots also at depths where no measurements were taken. Both procedures therefore aim at different objectives. A combined analysis could be possible, for example, by using marked point processes (Stoyan et al., 1995). We have restricted ourselves to the separate analysis, to show the quality, power and differences of the two approachers as clearly as possible.

An interesting feature of the depth-slice kriging procedure is that it can easily deal with non-stationarity in depth by considering each separately. This is of course not the only way to deal with non-stationary three-dimensional interpolation, and using a three-dimensional variogram may lead to a lower kriging 
variogram, and maybe even less smoothing than the depth-slices kriging proposed in this paper. We found the depth-slices kriging procedure attractive in its simplicity, and also appealing to the intuitive idea that the intrinsic hypothesis is easily applicable in planar space, but that occurrence of depth processes and irregular behavior of stains at several depths with abrupt changes that are difficult to assess makes it difficult to make any stationarity assumption at all.

The question is now to which extent our findings may contribute to issues of precision agriculture. A better understanding of the connections between the three different types of stains might give a clue towards better understanding of the movement of moisture containing nitrate and pesticides towards the groundwater. This study, though, is only a first step towards a full understanding of such behavior. For example, one might need a full pattern of all stains within a field to fully understand leaching of such moisture. That is clearly impossible to achieve. But observing and registering a clearly visible pattern of cracks may help to identify the main component of leaching channels, and further modelling of their relations with channels and vughs may then help to make a quantitative assessment of leaching. But at this stage, our primary objective was to better understand the nature of vughs, with some ideas on precision agriculture as the leading guide.

\section{Conclusions}

A spatial statistical point pattern analysis and a geostatistical image analysis are complementary. The point pattern analysis highlights elements of a soil considered as a medium to transport moisture, whereas geostatistics estimates occurrence of moisture at those depths where management decision may be taken. Both procedures have a limited validity only. Point patterns as analysed in this paper neglect the obvious positive support of the stains, in particular for cracks. However, the structure functions all elucidate this by increasing up to the average size of the stains. A geostatistical analysis did not consider the typical features of the individual stains. As a combination, though, a rather complete picture emerges, that may serve as a general approach to modelling blue-colored stains in soils.

The role of stains in soils is directly related to the movement of moisture. Blue-colored water spilled at the soil surface highlights those parts of the soil that are directly reached by moisture. Blue-colored moisture therefore serves as a proxy for moisture containing nitrate and pesticides. Obviously, larger sized cracks are the main transport channels, to which vughs and channels are related in a way described in this paper. The geostatistical procedure emphasizes those areas, even at unvisited depths, that are likely to be reached by moisture containing nitrate and pesticides, as well as the less sensitive ones. 


\section{Acknowledgements}

We gratefully acknowledge the assistance of Dr. Ben Gorte at Delft Technical University during the preparation of this manuscript.

\section{References}

Baddeley, A.J., 1999. Spatial sampling and censoring. In: Barndorff-Nielsen, O.E., Kendall, W.S., Van Lieshout, M.N.M. (Eds.), Stochastic Geometry, Likelihood and Computation. Monographs on Statistics and Applied Probability, vol. 80, Chapman \& Hall/CRC, London, pp. 37-78.

Baddeley, A.J., Gill, R.D., 1998. Kaplan-Meier estimators for interpoint distance distributions of spatial point processes. Ann. Stat. 25, 263-292.

Barnett, V., Payne, R., Steiner, V., 1995. Agricultural Sustainability: Economic, Environmental and Statistical Considerations. Wiley, Chichester, $278 \mathrm{pp}$.

Bartoli, F., Philippy, R., Doirisse, M., Niquet, S., Dubuit, K., 1991. Structure and self-similarity in silty and sandy soils: the fractal approach. J. Soil Sci. 42, 167-185.

Bartoli, F., Bird, N.R.A., Gomendy, V., Vivier, H., Niquet, S., 1999. The relation between silty soil structures and their mercury porosimetry curve counterparts: fractals and percolation. Eur. J. Soil Sci. 50, 9-22.

Beven, K., Germann, P., 1982. Macropores and water flow in soils. Water Resour. Res. 18, $1311-1325$.

Booltink, H.W.G., Hatano, R., Bouma, J., 1993. Measurement and simulation of bypass flow in a structured clay soil: a physico-morphological approach. J. Hydrol. 148, 149-168.

Bouma, J., Jongerius, A., Boersma, O., De Jager, A., Schoonderbeek, D., 1977. The function of different types of macropores during saturated flow through swelling soil horizons. Soil Sci. Soc. Am. J. 41, 945-950.

Capowicz, Y., Pierret, A., Daniel, O., Monestiez, P., 1998. 3D sjkeleton reconstructions of natural earthworm burrow systems using CAT scan images of soil cores. Biol. Fertil. Soils 27, 51-59.

Chadoeuf, J., Kretzschmar, A., Goulard, M., Smettem, K.R., 1994. Description of the spatial interaction between earthworm burrows and cracks at the soil surface. In: Ringroase-Voase, A.J., Humphreys, G.S. (Eds.), Soil Micromorphology: Studies in Management and Genesis. Proceedings of the Ninth International Working Meeting on Soil Micromorphology, Elsevier. Amsterdam, pp. 521-530.

CIBA, 1997. Precision Agriculture: Spatial and Temporal Variability of Environmental Quality. Wiley, Chichester.

Cressie, N.A.C., 1991. Statistics for Spatial Data. Wiley, New York, 900 pp.

Dagan, G., 1989. Flow and Transport in Porous Formations. Springer, Berlin, 465 pp.

Daniel, O., Kretzschmar, A., Capowicz, Y., Kohli, Y., Zeyer, J., 1997. Computer-assisted tomography of macroporosirty and its application to study the activity of the earthworm Aporrectodea nocturna. Eur. J. Soil Sci. 48, 727-737.

Debras, J.F., Faivre d'Arcier, F., Buffiere, M.J., Rieux, R., Simon, S., Kretzschmar, A., 1998. Does the global load in a pear orchard of psyllids and predators describe an equilibrium of predators/prey and does it permit a control strategy. First Transnational Workshop on Biological, Integrated and Rational Control: Status and Perspectives with Respect to Regional and European Experiences. Service National de la Protection des Vegetaux, Nord Pas-de-Calais, Loss-en-Gohelle, pp. 77-78.

Groffman, P.M., 1997. Ecological constraints on the ability of precision agriculture to improve the environmental performance of agricultural production systems (with discussion). In: Lake, 
J.V., Bock, G.R., Goode, J.A. (Eds.), Precision Agriculture: Spatial and Temporal Variability of Environmental Quality. Wiley, Chichester, pp. 52-67.

Hatano, R., Booltink, H.W.G., 1992. Using fractal dimensions of stained flow patterns in a clay soil to predict bypass flow. J. Hydrol. 135, 121-131.

Kaplan, E.L., Meier, P., 1958. Non-parametric estimation from incomplete observations. J. Am. Stat. Assoc. 53, 457-481.

Lotwick, H.W., Silverman, B.W., 1982. Methods for analysing spatial processes of several types of points. J. Roy. Statist. Soc. B 44, 406-413.

Matheron, G., 1973. The intrinsic random functions and their applications. Adv. Appl. Probab. 5, 439-468.

Pierret, A., Capowicz, Y., Moran, C.J., Kretschmar, A., 1999. X-ray computed tomography to quantify tree rooting spatial distributions. Geoderma 90, 307-326.

Ringrose-Voase, A.J., Bullock, P., 1984. The automatic recognition and measurement of soil pore types by image analysis and computer programs. J. Soil Sci. 35, 673-684.

Ripley, B.D., 1977. Modelling spatial patterns (with discussion). J. R. Stat. Soc. B 39, 172-212.

Serra, J., 1982. Image Analysis and Mathematical Morphology. Academic Press, London, 610 pp.

Soil Survey Staff, 1992. Keys to Soil Taxonomy. 5th edn. Pocahontas Press, Blacksburg, SMSS Technical Monograph Nr. 19.

Stein, A., 1994. Sustainable agriculture: a challenge for statistics. In: Talbort, M. (Ed.), Quantitative Methods for Sustainable Agriculture. Scottish Agricultural Statistics Service, Edinburgh, pp. $1-14$.

Stein, A., Hoosbeek, M.R., Sterk, G., 1997. Space-time statistics for decision support to smart farming (with discussion). In: Lake, J.V., Bock, G.R., Goode, J.A. (Eds.), Precision Agriculture: Spatial and Temporal Variability of Environmental Quality. Wiley, Chichester, pp. $120-133$.

Stein, A., Droogers, P., Booltink, H.W.G., 1998. Point processes and random sets for analyzing patterns of methylene blue coloured soil. Soil Tillage Res. 46, 273-288.

Stoyan, D., Kendall, W.S., Mecke, J., 1995. Stochastic Geometry and its Applications. 2nd edn. Wiley, Chichester.

Ten Kate, T.K., Van Balen, R., Smeulders, A.W.M., Groen, F.C.A., Den Boer, G.A., 1990. SCILAIM: a multi-level interactive image processing environment. Pattern Recognition Letters $11,429-441$.

Van Lieshout, M.N.M., Baddely, A.J., 1997. A nonparametric measure of interaction in point processes. Stat. Neerlandica 50, 344-361.

Van Lieshout, M.N.M., Baddeley, A.J., 1999. Indices of dependence between types in multivariate point patterns. Scand. J. Stat. 26, 511-532.

Vogel, H.J., Kretzschmar, A., 1996. Topological characterization of pore space in soil-sample preparation and digital image-processing. Geoderma 73, 23-38. 